QUARTERLY OF APPLIED MATHEMATICS

VOLUME LXIV, NUMBER 4

DECEMBER 2006, PAGES 695-710

S $0033-569 X(06) 01041-0$

Article electronically published on October 31, 2006

\title{
SCATTERING RELATIONS \\ FOR POINT-GENERATED DYADIC FIELDS IN TWO-DIMENSIONAL LINEAR ELASTICITY
}

\author{
BY \\ C. ATHANASIADIS (Department of Mathematics, University of Athens, Panepistimiopolis, GR 157 \\ 84 Athens, Greece), \\ V. SEVROGLOU (Department of Mathematics, University of Ioannina, GR 45110 Ioannina, \\ Greece), \\ AND \\ I. G. STRATIS (Department of Mathematics, University of Athens, Panepistimiopolis, GR 15784 \\ Athens, Greece)
}

\begin{abstract}
The problem of scattering of elastic waves by a bounded obstacle in twodimensional linear elasticity is considered. The scattering problems are presented in a dyadic form. An incident dyadic field generated by a point source is disturbed by a rigid body, a cavity, or a penetrable obstacle. General scattering theorems are proved, relating the far-field patterns due to scattering of waves from a point source set up in either of two different locations. The most general reciprocity theorem is established, and mixed scattering relations are also proved. Finally, a relation between the incident and the scattered wave which refers to the mechanism of energy transfer of the scatterer, the so-called optical theorem, is established.
\end{abstract}

1. Introduction. Scattering theory deals with the propagation of a known incident wave field and with the disturbance that a given obstacle causes upon this propagation. Many researchers have provided solutions, both analytical and numerical, for the scattering of incident plane-wave fields. The theory of scattering of point-generated waves is not equally extended. In this area there are some general results that connect the solutions of two related problems. The most familiar of these is reciprocity: the scattered field

Received January 19, 2006.

2000 Mathematics Subject Classification. Primary 74J20; Secondary 74B05.

Key words and phrases. Dyadic scattering, point sources, scattering relations.

The authors acknowledge partial financial support from EPEAEK II ("Pythagoras II" research fellowships, project title "Mathematical Analysis of Wave Propagation in Chiral Electromagnetic and Elastic Media", University of Athens).

E-mail address: cathan@math.uoa.gr

E-mail address: bsevro@cc.uoi.gr

E-mail address: istratis@math.uoa.gr

(C)2006 Brown University Reverts to public domain 28 years from publication 
at $A$ due to a source at $B$ is simply related to the scattered field at $B$ due to a source at $A$. There are also internal relations within a single problem. A well-known example is the optical theorem for scattering of plane waves: it relates the far-field pattern in the forward direction to a certain integral of the far-field pattern over all directions.

In this paper, we derive some general relations for scattering of elastic waves emanating from point sources in two dimensions. Thus, we relate one problem with a point source at $A$ to a similar problem with a point source at $B$, and we prove the general scattering theorem. By setting $A=B$ and then letting $A$ recede to infinity, we recover the optical theorem. If we keep $A$ fixed and let $B$ recede to infinity, we obtain so-called mixed scattering theorems, relating plane-wave incidence to point-source incidence. An example of these is the mixed reciprocity theorem, which has found much use recently in methods for solving inverse scattering problems [25].

Clebsch considered the scattering of elastic waves from a point source by a rigid sphere 140 years ago, a decade before Lord Rayleigh published his solution for the scattering of a plane sound wave by a sphere.

The revival of interest in problems related to point-generated wave fields happened for several reasons. One is due to the variety of applications coming from the theory of composite materials and of acoustic emission, from the theoretical analysis of biological studies at the cell level, from nondestructive testing and evaluation, from geophysics, from modelling in medicine and the health sciences, and from scattering problems connected to environmental data analysis. Another reason is due to the fact that a point-source field is more easily realizable in a laboratory.

For the case of acoustic scattering, there has been recent work on incident waves generated by a point source in the vicinity of a scatterer; see, for example, [4], [5], and the references therein (especially the work by Dassios and his co-workers).

For the case of electromagnetic scattering, similar problems regarding point-source excitation have been studied in [5], 6], 7].

Regarding elasticity (versus electromagnetics), we note that while electromagnetic waves propagate at the same speed, the elastic wave consists of two independent parts (transverse and longitudinal) that are travelling with two different velocities. Our aim in the present paper is to extend the ideas in [5] in the two-dimensional elastic case.

Scattering in 3D linear elasticity for an incident plane vector or a dyadic field has been studied in [11, 12] and [15]-[17]. Different aspects of propagation and scattering problems in 2D linear elasticity are considered in [1]-3], 19], 21]-24, and [26]-[28]. Scattering for point sources in 2D linear elasticity is considered in [21, [22], [31].

We organize our paper as follows. In Section 2 we formulate the dyadic scattering problem for an incident elastic wave due to a source located at a point with position vector a. The cases of a rigid body, a cavity and a penetrable obstacle are considered and the dyadic forms of the longitudinal and transverse far-field patterns are given. In Section 3 the general scattering theorem is proved for the three cases above. In Section 4 reciprocity relations are established, while in Section 5 mixed scattering relations are proved, relating plane-wave incidence to point-source incidence. Finally, in Section 6, the optical theorem, which is a relation between the scattering amplitude and the total energy that the scatterer removes from the incident field, is established. 
2. Dyadic formulation of the problem. Let $B_{i}$ denote an open, bounded and simply connected subset of $\mathbb{R}^{2}$ with boundary $\partial B_{i}$, which is assumed to be a bounded Lyapunov surface. The set $B_{i}$ will be referred to as the scatterer. $B_{i}$ is filled with a material specified by the Lamé constants $\lambda_{i}, \mu_{i}$ and mass density $\rho_{i}$. The exterior domain $B_{e}=\mathbb{R}^{2} \backslash \bar{B}_{i}$, where $\bar{B}_{i}=B_{i} \cup \partial B_{i}$, is characterized by the Lamé constants $\lambda_{e}$ and $\mu_{e}$ and mass density $\rho_{e}$. The interior and exterior regions $B_{\alpha}$, denoted $\alpha=i, e$, are occupied by homogeneous and isotropic elastic materials, with the Lamé constants $\lambda_{\alpha}, \mu_{\alpha}$, respectively, that are assumed to satisfy the strong ellipticity conditions $\mu_{\alpha}>$ $0, \lambda_{\alpha}+2 \mu_{\alpha}>0$, in order for the media to sustain longitudinal as well as transverse waves.

In what follows we consider the scattering problems in a dyadic formulation. We choose this alternative method to study the problems due to the dyadic nature of the fundamental Green's function. It is worth mentioning that, as Twersky [30] pointed out for electromagnetic waves, the dyadic scattering problem - because of its higher symmetry - is easier than the corresponding vector scattering problem. This is so, because in the vector problem we need to specify both the polarization as well as the propagation vector, while in the dyadic problem the propagation vector alone suffices to specify the incident field. The dyadic formulation of elastic scattering problems in two dimensions can be found in 26,27 .

Assuming time-spectral decomposition, $\mathbf{U}(\mathbf{r}, t)=\mathbf{u}(\mathbf{r}) \exp \{-i \omega t\}$, the governing equation of linearized elasticity that the displacement field satisfies is the well-known spectral Navier equation

$$
c_{s}^{2} \Delta \mathbf{u}(\mathbf{r})+\left(c_{p}^{2}-c_{s}^{2}\right) \nabla \nabla \cdot \mathbf{u}(\mathbf{r})+\omega^{2} \mathbf{u}(\mathbf{r})=\mathbf{0},
$$

where $\omega$ is the angular frequency (denotes the Fourier dual variable of $t$ ), and $c_{p}, c_{s}$ are the phase velocities of the longitudinal and the transverse wave, respectively, given by $c_{p}=\sqrt{(\lambda+2 \mu) / \rho}, \quad c_{s}=\sqrt{\mu / \rho}$. The phase velocities are connected with the angular frequency $\omega$, via the relations $\omega=k_{p} c_{p}=k_{s} c_{s}$, where $k_{p}=2 \pi / \lambda_{p}$ and $k_{s}=2 \pi / \lambda_{s}$ are the wave numbers for the longitudinal and the transverse waves, respectively, and $\lambda_{p}, \lambda_{s}$ are the corresponding wavelengths.

Now adopting dyadic scattering, we generalize the above formulation into a dyadic setting. In what follows, properties of dyadics will be used, and an excellent source for dyadic formulas is the book by Tai 29 . So, let $\widetilde{\mathbf{u}}(\mathbf{r})$ be a dyadic field (" " (the overtilde) will denote dyadic fields in this paper) and $\mathbf{c}$ any constant vector. Then, if the vector field $\mathbf{u}(\mathbf{r})=\widetilde{\mathbf{u}}(\mathbf{r}) \cdot \mathbf{c}$ solves the spectral Navier Equation (2.1), then $\widetilde{\mathbf{u}}(\mathbf{r})$ solves the following dyadic version of (2.1):

$$
c_{s}^{2} \Delta \widetilde{\mathbf{u}}(\mathbf{r})+\left(c_{p}^{2}-c_{s}^{2}\right) \nabla \nabla \cdot \widetilde{\mathbf{u}}(\mathbf{r})+\omega^{2} \widetilde{\mathbf{u}}(\mathbf{r})=\widetilde{\mathbf{0}} .
$$

For the displacement field $\widetilde{\mathbf{u}}(\mathbf{r})$, we can apply the well-known Helmholtz decomposition 20]: if $\widetilde{\mathbf{u}}^{p}(\mathbf{r})$ is the longitudinal part (dyadic P-wave) and $\widetilde{\mathbf{u}}^{s}(\mathbf{r})$ the transverse part (dyadic S-wave), then the displacement field $\widetilde{\mathbf{u}}$ is decomposed as $\widetilde{\mathbf{u}}(\mathbf{r})=\widetilde{\mathbf{u}}^{p}(\mathbf{r})+\widetilde{\mathbf{u}}^{s}(\mathbf{r})$.

The differential equations that the displacement fields satisfy in the regions $B_{\alpha}, \alpha=$ $e, i$, can be written as (an alternative form of equation (2.1))

$$
\left(\Delta_{\alpha}^{*}+\rho_{\alpha} \omega^{2}\right) \widetilde{\mathbf{u}}_{\alpha}(\mathbf{r})=\widetilde{\mathbf{0}}, \quad \mathbf{r} \in B_{\alpha},
$$


where the fields $\widetilde{\mathbf{u}}_{\alpha}(\mathbf{r})$ represent the interior $(\alpha=i)$ and exterior $(\alpha=e)$ displacement fields, respectively, and $\Delta_{\alpha}^{*}=\mu_{\alpha} \Delta+\left(\lambda_{\alpha}+\mu_{\alpha}\right) \nabla \nabla$.

We also define the surface stress operator

$$
T_{\alpha}^{(\mathbf{r})}=2 \mu_{\alpha} \hat{\mathbf{n}}_{\mathbf{r}} \cdot \nabla+\lambda_{\alpha} \hat{\mathbf{n}}_{\mathbf{r}} \nabla \cdot+\mu_{\alpha} \hat{\mathbf{n}}_{\mathbf{r}} \times \nabla \times,
$$

where $\hat{\mathbf{n}}_{\mathbf{r}}$ stands for the outward unit normal vector on $\partial B_{i}$ at the point $\mathbf{r}$, and the superscript (which will be omitted from now on) denotes the action of the differential operator on the indicated variable.

We irradiate our object by an incident elastic wave due to a source located at a point with position vector $\mathbf{a}$, i.e.,

$$
\begin{aligned}
\widetilde{\mathbf{u}}_{a}^{i n c}(\mathbf{r})= & -\frac{i}{4 \omega^{2}} \nabla_{\mathbf{r}} \nabla_{\mathbf{r}} H_{0}^{(1)}\left(k_{p}|\mathbf{r}-\mathbf{a}|\right) \\
& +\frac{i}{4 \omega^{2}}\left(\nabla_{\mathbf{r}} \nabla_{\mathbf{r}}+k_{s}^{2} \widetilde{\mathbf{I}}\right) H_{0}^{(1)}\left(k_{s}|\mathbf{r}-\mathbf{a}|\right), \quad \mathbf{r} \neq \mathbf{a},
\end{aligned}
$$

where $\widetilde{\mathbf{I}}$ is the identity dyadic, and $H_{0}^{(1)}(z)$ is the Hankel function of first kind and zero order. This is actually similar to the fundamental solution with a singularity at the point a, [19.

Now let $a=|\mathbf{a}| \rightarrow \infty$. Then the incident point-source field given by (2.5) reduces to a dyadic plane wave with direction of propagation - $\hat{\mathbf{a}}$. Indeed, using the asymptotic behaviour of the Hankel function of the first kind and zero order, 20, we obtain

$$
\widetilde{\mathbf{u}}^{i n c}(\mathbf{r} ;-\hat{\mathbf{a}})=A_{p}(\hat{\mathbf{a}} \otimes \hat{\mathbf{a}}) e^{-i k_{p} \mathbf{r} \cdot \hat{\mathbf{a}}}+A_{s}(\widetilde{\mathbf{I}}-\hat{\mathbf{a}} \otimes \hat{\mathbf{a}}) e^{-i k_{s} \mathbf{r} \cdot \hat{\mathbf{a}}},
$$

where " $\otimes$ " is the juxtaposition between two vectors (this gives a dyadic) and $A_{p}, A_{s}$ are constant amplitudes given by

$$
A_{p}:=\frac{1}{\lambda+2 \mu} \frac{(1+i) e^{i k_{p} a}}{4 \sqrt{\pi k_{p} a}} \quad \text { and } \quad A_{s}:=\frac{1}{\mu} \frac{(1+i) e^{i k_{s} a}}{4 \sqrt{\pi k_{s} a}} .
$$

Due to the point-source incident field at $\mathbf{a}$, the corresponding component of the scattered field is denoted by $\widetilde{\mathbf{u}}_{a}^{s c t}$. Then the total field $\widetilde{\mathbf{u}}_{a}^{\text {tot }}$ in the exterior $B_{e}$ of the scatterer is given by

$$
\widetilde{\mathbf{u}}_{a}^{t o t}(\mathbf{r})=\widetilde{\mathbf{u}}_{a}^{i n c}(\mathbf{r})+\widetilde{\mathbf{u}}_{a}^{s c t}(\mathbf{r}),
$$

where the incident, the scattered and the total field satisfy Eq. (2.2). We consider the following boundary conditions: for a rigid scatterer,

$$
\widetilde{\mathbf{u}}_{a}^{t o t}(\mathbf{r})=\widetilde{\mathbf{0}}, \quad \mathbf{r} \in \partial B_{i} ;
$$

for a cavity, the action of the surface stress operator on the displacement field must be zero, i.e.,

$$
T_{e} \widetilde{\mathbf{u}}_{a}^{t o t}(\mathbf{r})=\widetilde{\mathbf{0}}, \quad \mathbf{r} \in \partial B_{i} ;
$$

while for the penetrable case,

$$
\begin{aligned}
\widetilde{\mathbf{u}}_{a}^{t o t}(\mathbf{r}) & =\widetilde{\mathbf{u}}_{a}^{i n t}(\mathbf{r}), & & \mathbf{r} \in \partial B_{i}, \\
T_{e} \widetilde{\mathbf{u}}_{a}^{t o t}(\mathbf{r}) & =T_{i} \widetilde{\mathbf{u}}_{a}^{i n t}(\mathbf{r}), & & \mathbf{r} \in \partial B_{i},
\end{aligned}
$$

where $\widetilde{\mathbf{u}}_{a}^{i n t}$ is the interior elastic field in $B_{i}$. 
For the well-posedness of these problems, the well-known radiation conditions due to Kupradze should also be satisfied by the scattered field in the following dyadic form:

$$
\lim _{r \rightarrow \infty} \widetilde{\mathbf{u}}_{a}^{s c t, \beta}=\widetilde{\mathbf{0}}, \quad \lim _{r \rightarrow \infty} \sqrt{r}\left(\frac{\partial \widetilde{\mathbf{u}}_{a}^{s c t, \beta}}{\partial r}-i k_{\beta} \widetilde{\mathbf{u}}_{a}^{s c t, \beta}\right)=\widetilde{\mathbf{0}}, \quad \beta=p, s .
$$

Using the dyadic formulation [26, exploiting Betti's formulae, and through asymptotic analysis, we obtain

$$
\widetilde{\mathbf{u}}_{a}^{s c t}(\mathbf{r})=\widetilde{\mathbf{g}}_{a}^{r}(\hat{\mathbf{r}}) \frac{e^{i k_{p} r}}{\sqrt{r}}+\widetilde{\mathbf{g}}_{a}^{t}(\hat{\mathbf{r}}) \frac{e^{i k_{s} r}}{\sqrt{r}}+O\left(r^{-3 / 2}\right), \quad r \rightarrow \infty,
$$

uniformly with respect to $\hat{\mathbf{r}}=\frac{\mathbf{r}}{r} \in \Omega$, where $\Omega$ is the unit circle in $\mathbb{R}^{2}$. The coefficients of the terms $\frac{e^{i k_{\beta^{r}}}}{\sqrt{r}}$ are the corresponding dyadic far-field patterns (defined on $\Omega$ ) and are known as the longitudinal and the transverse far-field patterns, respectively. They are given by

$$
\begin{aligned}
& \widetilde{\mathbf{g}}_{a}^{r}(\hat{\mathbf{r}})=(\hat{\mathbf{r}} \otimes \hat{\mathbf{r}}) \cdot\left[\frac{1+i}{4 c_{p}^{2} \sqrt{\pi k_{p}}} \widetilde{\mathbf{L}}_{p}(\hat{\mathbf{r}})+\frac{1-i}{4} \sqrt{\frac{k_{p}}{\pi}} \widetilde{\mathbf{H}}_{p}(\hat{\mathbf{r}})\right], \\
& \widetilde{\mathbf{g}}_{a}^{t}(\hat{\mathbf{r}})=(\widetilde{\mathbf{I}}-\hat{\mathbf{r}} \otimes \hat{\mathbf{r}}) \cdot\left[\frac{1+i}{4 c_{s}^{2} \sqrt{\pi k_{s}}} \widetilde{\mathbf{L}}_{s}(\hat{\mathbf{r}})+\frac{1-i}{4} \sqrt{\frac{k_{s}}{\pi}} \widetilde{\mathbf{H}}_{s}(\hat{\mathbf{r}})\right],
\end{aligned}
$$

where

$$
\begin{aligned}
\widetilde{\mathbf{L}}_{p}(\hat{\mathbf{r}}) & =-\int_{\partial B_{i}} T \widetilde{\mathbf{u}}_{a}^{t o t}\left(\mathbf{r}^{\prime}\right) e^{-i k_{p} \mathbf{r}^{\prime} \cdot \hat{\mathbf{r}}} d s\left(\mathbf{r}^{\prime}\right), \\
\widetilde{\mathbf{L}}_{s}(\hat{\mathbf{r}}) & =-\int_{\partial B_{i}} T \widetilde{\mathbf{u}}_{a}^{t o t}\left(\mathbf{r}^{\prime}\right) e^{-i k_{s} \mathbf{r}^{\prime} \cdot \hat{\mathbf{r}}} d s\left(\mathbf{r}^{\prime}\right), \\
\widetilde{\mathbf{H}}_{p}(\hat{\mathbf{r}}) & =\int_{\partial B_{i}}\left(\hat{\mathbf{n}}^{\prime} \cdot \frac{\lambda \widetilde{\mathbf{I}}+2 \mu \hat{\mathbf{r}} \otimes \hat{\mathbf{r}}}{\lambda+2 \mu} \otimes \hat{\mathbf{r}}\right) \cdot \widetilde{\mathbf{u}}_{a}^{t o t}\left(\mathbf{r}^{\prime}\right) e^{-i k_{p} \mathbf{r}^{\prime} \cdot \hat{\mathbf{r}}} d s\left(\mathbf{r}^{\prime}\right), \\
\widetilde{\mathbf{H}}_{s}(\hat{\mathbf{r}}) & =\int_{\partial B_{i}}\left(\left[\left(\hat{\mathbf{n}}^{\prime} \cdot \hat{\mathbf{r}}\right) \hat{\mathbf{r}}^{\perp}+\left(\hat{\mathbf{n}}^{\prime} \cdot \hat{\mathbf{r}}^{\perp}\right) \hat{\mathbf{r}}\right] \otimes \hat{\mathbf{r}}^{\perp}\right) \cdot \widetilde{\mathbf{u}}_{a}^{t o t}\left(\mathbf{r}^{\prime}\right) e^{-i k_{s} \mathbf{r}^{\prime} \cdot \hat{\mathbf{r}}} d s\left(\mathbf{r}^{\prime}\right) .
\end{aligned}
$$

The dyadics $\hat{\mathbf{r}} \otimes \hat{\mathbf{r}}$ and $\widetilde{\mathbf{I}}-\hat{\mathbf{r}} \otimes \hat{\mathbf{r}}$ represent the radial and tangential behaviour of the longitudinal and transverse parts, respectively, of the scattered field far away from the scatterer.

We recall again that the scatterer is irradiated by an incident point-source field of the form (2.5). In that case, when $r \rightarrow \infty$, the following form for (2.5) holds:

$$
\begin{aligned}
\widetilde{\mathbf{u}}_{a}^{i n c}(\mathbf{r})= & B_{p}(\hat{\mathbf{r}} \otimes \hat{\mathbf{r}}) \frac{e^{i k_{p} r}}{\sqrt{r}} e^{-i k_{p} \mathbf{a} \cdot \hat{\mathbf{r}}} \\
& +B_{s}(\widetilde{\mathbf{I}}-\hat{\mathbf{r}} \otimes \hat{\mathbf{r}}) \frac{e^{i k_{s} r}}{\sqrt{r}} e^{-i k_{s} \mathbf{a} \cdot \hat{\mathbf{r}}}+O\left(r^{-3 / 2}\right), \quad r \rightarrow \infty
\end{aligned}
$$

where $B_{p}=A_{p} \sqrt{a} e^{-i k_{p} a}, B_{s}=A_{s} \sqrt{a} e^{-i k_{s} a}$ and $A_{p}, A_{s}$ are given by (2.7). 
3. The general scattering theorem. In this section scattering relations for 2Dpoint-generated elastic waves are established, with a similar procedure of related 3D results in acoustics and electromagnetics [4-7].

In what follows, we introduce Twersky's notation

$$
[\widetilde{\mathbf{u}}(\mathbf{r}), \widetilde{\mathbf{v}}(\mathbf{r})]_{\partial B_{i}}:=\int_{\partial B_{i}}\left[(T \widetilde{\mathbf{v}}(\mathbf{r}))^{\top} \cdot \widetilde{\mathbf{u}}(\mathbf{r})-\widetilde{\mathbf{v}}(\mathbf{r})^{\top} \cdot T \widetilde{\mathbf{u}}(\mathbf{r})\right] d s(\mathbf{r}),
$$

where "." is the dot product between two dyadics (this gives a dyadic) and " $T$ " denotes transposition. The subscript " $\partial B_{i}$ " in the above notation will be omitted from now on, so we use $[\widetilde{\mathbf{u}}, \widetilde{\mathbf{v}}]$ instead of $[\widetilde{\mathbf{u}}, \widetilde{\mathbf{v}}]_{\partial B_{i}}$.

Consider now two point sources $\mathbf{a}$ and $\mathbf{b}$ with corresponding scattered fields $\widetilde{\mathbf{u}}_{a}^{\text {sct }}$ and $\widetilde{\mathbf{u}}_{b}^{s c t}$, respectively. In addition, let $\Omega_{a, \varepsilon}=\left\{\mathbf{r} \in \mathbb{R}^{2}: \varepsilon=|\mathbf{r}-\mathbf{a}|\right\}$ be a small circle centered at $\mathbf{a}$, and $\Omega_{r}$ be a large circle of radius $r$ surrounding $\bar{B}_{i}$, the point source $\mathbf{b}$ and $\Omega_{a, \varepsilon}$. In the sequel the overbar denotes complex conjugation. Then, we prove the following:

LEmma 3.1. Let $\widetilde{\mathbf{u}}_{a}^{i n c}$ be an incident point-source field at a. For a given point source at $\mathbf{b}$, with corresponding scattered field $\widetilde{\mathbf{u}}_{b}^{s c t}$ and longitudinal and transverse far-field patterns $\widetilde{\mathbf{g}}_{b}^{r}$ and $\widetilde{\mathbf{g}}_{b}^{t}$, respectively, we have

$$
\begin{aligned}
& \lim _{r \rightarrow \infty}\left[\overline{\widetilde{\mathbf{u}}_{a}^{i n c}}, \widetilde{\mathbf{u}}_{b}^{s c t}\right]_{\Omega_{r}}=\frac{1+i}{2} \sqrt{\frac{k_{p}}{\pi}} \int_{\Omega}\left(\widetilde{\mathbf{g}}_{b}^{r}(\widehat{\mathbf{r}})\right)^{\top} \cdot(\hat{\mathbf{r}} \otimes \hat{\mathbf{r}}) e^{i k_{p} \mathbf{a} \cdot \hat{\mathbf{r}}} d s(\hat{\mathbf{r}}) \\
& +\frac{1+i}{2} \sqrt{\frac{k_{s}}{\pi}} \int_{\Omega}\left(\widetilde{\mathbf{g}}_{b}^{t}(\hat{\mathbf{r}})\right)^{\top} \cdot(\widetilde{\mathbf{I}}-\hat{\mathbf{r}} \otimes \hat{\mathbf{r}}) e^{i k_{s} \mathbf{a} \cdot \hat{\mathbf{r}}} d s(\hat{\mathbf{r}}), \\
& \lim _{\varepsilon \rightarrow 0}\left[\overline{\widetilde{\mathbf{u}}_{a}^{i n c}}, \widetilde{\mathbf{u}}_{b}^{s c t}\right]_{\Omega_{a, \varepsilon}}=\left(\widetilde{\mathbf{u}}_{b}^{s c t}(\mathbf{a})\right)^{\top} \cdot(\hat{\mathbf{a}} \otimes \hat{\mathbf{a}})+\frac{\lambda+\mu}{\mu}\left(\widetilde{\mathbf{u}}_{b}^{s c t}(\mathbf{a})\right)^{\top} \cdot(\widetilde{\mathbf{I}}-\hat{\mathbf{a}} \otimes \hat{\mathbf{a}}) .
\end{aligned}
$$

Proof. Using equations (2.13) and (2.20) we can calculate the dyadic fields $T \overline{\widetilde{\mathbf{u}}_{a}^{\text {inc }}(\mathbf{r})}$ and $T \widetilde{\mathbf{u}}_{b}^{s c t}(\mathbf{r})$. After lengthy calculations, we arrive at

$$
\begin{aligned}
& T \overline{\widetilde{\mathbf{u}}_{a}^{i n c}(\mathbf{r})}=- \frac{1+i}{4} \sqrt{\frac{k_{p}}{\pi}} \frac{e^{-i k_{p} r}}{\sqrt{r}}(\hat{\mathbf{r}} \otimes \hat{\mathbf{r}}) e^{i k_{p} \mathbf{a} \cdot \hat{\mathbf{r}}} \\
&-\frac{1+i}{4} \sqrt{\frac{k_{s}}{\pi}} \frac{e^{-i k_{s} r}}{\sqrt{r}}(\widetilde{\mathbf{I}}-\hat{\mathbf{r}} \otimes \hat{\mathbf{r}}) e^{i k_{s} \mathbf{a} \cdot \hat{\mathbf{r}}}+O\left(r^{-3 / 2}\right), \quad r \rightarrow \infty, \\
& T \widetilde{\mathbf{u}}_{b}^{s c t}(\mathbf{r})=i k_{p}(\lambda+2 \mu) \widetilde{\mathbf{g}}_{b}^{r}(\hat{\mathbf{r}}) \frac{e^{i k_{p} r}}{\sqrt{r}}+i k_{s} \mu \widetilde{\mathbf{g}}_{b}^{t}(\hat{\mathbf{r}}) \frac{e^{i k_{s} r}}{\sqrt{r}}+O\left(r^{-3 / 2}\right), \quad r \rightarrow \infty .
\end{aligned}
$$

Combining (3.4) and (3.5) we have the following relation:

$$
\begin{aligned}
& \int_{\Omega_{r}}\left(\left(T \widetilde{\mathbf{u}}_{b}^{s c t}(\mathbf{r})\right)^{\top} \cdot \overline{\widetilde{\mathbf{u}}_{a}^{i n c}(\mathbf{r})}-\left(\widetilde{\mathbf{u}}_{b}^{s c t}(\mathbf{r})\right)^{\top} \cdot T \overline{\widetilde{\mathbf{u}}_{a}^{i n c}(\mathbf{r})}\right) d s(\mathbf{r}) \\
& =\frac{1+i}{2} \sqrt{\frac{k_{p}}{\pi}} \int_{\Omega}\left(\widetilde{\mathbf{g}}_{b}^{r}(\hat{\mathbf{r}})\right)^{\top} \cdot(\hat{\mathbf{r}} \otimes \hat{\mathbf{r}}) e^{i k_{p} \mathbf{a} \cdot \hat{\mathbf{r}}} d s(\hat{\mathbf{r}}) \\
& +\frac{1+i}{2} \sqrt{\frac{k_{s}}{\pi}} \int_{\Omega}\left(\widetilde{\mathbf{g}}_{b}^{t}(\hat{\mathbf{r}})\right)^{\top} \cdot(\widetilde{\mathbf{I}}-\hat{\mathbf{r}} \otimes \hat{\mathbf{r}}) e^{i k_{s} \mathbf{a} \cdot \hat{\mathbf{r}}} d s(\hat{\mathbf{r}}),
\end{aligned}
$$

and hence, relation (3.2) is proved. 
For Eq. (3.3) we have to determine $\widetilde{\mathbf{u}}_{a}^{i n c}(\mathbf{r})$ and $T \overline{\widetilde{\mathbf{u}}_{a}^{\text {inc }}(\mathbf{r})}$ on the small circle $\Omega_{a, \varepsilon}$. Taking into account the recurrence and differential relations for $Z_{m}(x)$, [8], where $Z_{m}$ can be any of $H_{0}^{(1)}, H_{1}^{(1)}$ or $H_{2}^{(1)}$, and after some calculations, relation (2.5) can be written as

$$
\begin{aligned}
\widetilde{\mathbf{u}}_{a}^{i n c}(\mathbf{r})= & \frac{i}{4 \omega^{2}}\left(k_{p}^{2} H_{0}^{(1)}\left(k_{p} \varepsilon\right)-k_{s}^{2} H_{0}^{(1)}\left(k_{s} \varepsilon\right)\right) \hat{\mathbf{R}} \otimes \hat{\mathbf{R}} \\
& -\frac{i}{2 \omega^{2} \varepsilon}\left(k_{p} H_{1}^{(1)}\left(k_{p} \varepsilon\right)-k_{s} H_{1}^{(1)}\left(k_{s} \varepsilon\right)\right) \hat{\mathbf{R}} \otimes \hat{\mathbf{R}} \\
& +\frac{i}{4 \omega^{2} \varepsilon}\left(k_{p} H_{1}^{(1)}\left(k_{p} \varepsilon\right)-k_{s} H_{1}^{(1)}\left(k_{s} \varepsilon\right)\right) \widetilde{\mathbf{I}} \\
& +\frac{i}{4 \mu} H_{0}^{(1)}\left(k_{s} \varepsilon\right) \widetilde{\mathbf{I}},
\end{aligned}
$$

where $\mathbf{R}=\mathbf{r}-\mathbf{a}, \varepsilon=|\mathbf{r}-\mathbf{a}|$ and $\hat{\mathbf{R}}=\frac{\mathbf{r}-\mathbf{a}}{|\mathbf{r}-\mathbf{a}|}$. The above formulation (3.7) is very convenient in the analysis of the singular behaviour of $\widetilde{\mathbf{u}}_{a}^{i n c}$ as $\varepsilon \rightarrow 0$. With the aid of the mean value theorem and using the asymptotic behaviour of $\frac{i}{4} H_{0}^{(1)}\left(k_{\beta} \varepsilon\right)$ [10, p. 124], and $H_{m}^{(1)}\left(k_{\beta} \varepsilon\right),[8]$, near the origin, i.e.,

$$
\begin{aligned}
\frac{i}{4} H_{0}^{(1)}\left(k_{\beta} \varepsilon\right) & \cong \frac{1}{2 \pi} \ln \frac{1}{\varepsilon}+\frac{i}{4}-\frac{1}{2 \pi} \ln \frac{k_{\beta}}{2}-\frac{C}{2 \pi}, \quad \varepsilon \rightarrow 0, \\
H_{m}^{(1)}\left(k_{\beta} \varepsilon\right) & \cong \frac{\left(k_{\beta} \varepsilon\right)^{m}}{2^{m} m !}-i \frac{2^{m}(m-1) !}{\pi\left(k_{\beta} \varepsilon\right)^{m}}, \quad m=1,2, \quad \varepsilon \rightarrow 0,
\end{aligned}
$$

where $C$ denotes Euler's constant, we establish the assertion.

For two point sources with position vectors $\mathbf{a}$ and $\mathbf{b}$, we introduce the following dyadic far-field pattern generators:

$$
\begin{aligned}
\widetilde{\mathbf{G}}_{b}^{r}(\mathbf{a})= & \frac{(1-i) \sqrt{k_{p}}}{4} \int_{\Omega}\left(\widetilde{\mathbf{g}}_{b}^{r}(\widehat{\mathbf{r}})\right)^{\top} \cdot(\hat{\mathbf{r}} \otimes \hat{\mathbf{r}}) e^{i k_{p} \mathbf{a} \cdot \hat{\mathbf{r}}} d s(\hat{\mathbf{r}}) \\
& +\frac{i \sqrt{\pi}}{2}\left(\widetilde{\mathbf{u}}_{b}^{s c t}(\mathbf{a})\right)^{\top} \cdot(\hat{\mathbf{a}} \otimes \hat{\mathbf{a}}), \\
\widetilde{\mathbf{G}}_{b}^{t}(\mathbf{a})= & \frac{(1-i) \sqrt{k_{s}}}{4} \int_{\Omega}\left(\widetilde{\mathbf{g}}_{b}^{t}(\hat{\mathbf{r}})\right)^{\top} \cdot(\widetilde{\mathbf{I}}-\hat{\mathbf{r}} \otimes \hat{\mathbf{r}}) e^{i k_{s} \mathbf{a} \cdot \hat{\mathbf{r}}} d s(\hat{\mathbf{r}}) \\
& +\frac{i \sqrt{\pi}}{2} \frac{\lambda+\mu}{\mu}\left(\widetilde{\mathbf{u}}_{b}^{s c t}(\mathbf{a})\right)^{\top} \cdot(\widetilde{\mathbf{I}}-\hat{\mathbf{a}} \otimes \hat{\mathbf{a}}) .
\end{aligned}
$$

We now establish the general dyadic scattering theorem for point sources.

TheOrem 3.2. Assume two point-source locations $\mathbf{a}$ and $\mathbf{b}$ in $B_{e}$. Then the following relation holds:

$$
\begin{aligned}
& \widetilde{\mathbf{G}}_{b}^{r}(\mathbf{a})+\left(\overline{\widetilde{\mathbf{G}}_{a}^{r}(\mathbf{b})}\right)^{\top}+\widetilde{\mathbf{G}}_{b}^{t}(\mathbf{a})+\left(\overline{\widetilde{\mathbf{G}}_{a}^{t}(\mathbf{b})}\right)^{\top} \\
& =-k_{p}(\lambda+2 \mu) \sqrt{\pi} \int_{\Omega}\left(\widetilde{\mathbf{g}}_{b}^{r}(\widehat{\mathbf{r}})\right)^{\top} \cdot \overline{\widetilde{\mathbf{g}}_{a}^{r}(\widehat{\mathbf{r}})} d s(\hat{\mathbf{r}})-k_{s} \mu \sqrt{\pi} \int_{\Omega}\left(\widetilde{\mathbf{g}}_{b}^{t}(\widehat{\mathbf{r}})\right)^{\top} \cdot \overline{\widetilde{\mathbf{g}}_{a}^{t}(\widehat{\mathbf{r}})} d s(\hat{\mathbf{r}}) .
\end{aligned}
$$


Proof. Since $\widetilde{\mathbf{u}}_{\gamma}^{\text {tot }}=\widetilde{\mathbf{u}}_{\gamma}^{\text {inc }}+\widetilde{\mathbf{u}}_{\gamma}^{s c t}, \gamma=a, b$, the bilinearity of $[\cdot, \cdot]$ yields

$$
\left[\overline{\widetilde{\mathbf{u}}_{a}^{t o t}}, \widetilde{\mathbf{u}}_{b}^{t o t}\right]=\left[\overline{\widetilde{\mathbf{u}}_{a}^{i n c}}, \widetilde{\mathbf{u}}_{b}^{i n c}\right]+\left[\overline{\widetilde{\mathbf{u}}_{a}^{i n c}}, \widetilde{\mathbf{u}}_{b}^{s c t}\right]+\left[\overline{\widetilde{\mathbf{u}}_{a}^{s c t}}, \widetilde{\mathbf{u}}_{b}^{i n c}\right]+\left[\overline{\widetilde{\mathbf{u}}_{a}^{s c t}}, \widetilde{\mathbf{u}}_{b}^{s c t}\right] .
$$

For all three types of boundary conditions (rigid scatterer, cavity, penetrable obstacle), we can easily get

$$
\left[\overline{\widetilde{\mathbf{u}}_{a}^{t o t}}, \widetilde{\mathbf{u}}_{b}^{t o t}\right]=\widetilde{\mathbf{0}} .
$$

We now calculate each term of the right-hand side of (3.13). In particular, the first term is vanishing since, by the third Betti's formula and the fact that $\widetilde{\mathbf{u}}_{a}^{i n c}$ and $\widetilde{\mathbf{u}}_{b}^{i n c}$ are regular solutions of the Navier equation (2.3) in $B_{i}$, we can obtain

$$
\left[\overline{\widetilde{\mathbf{u}}_{a}^{i n c}}, \widetilde{\mathbf{u}}_{b}^{i n c}\right]=\widetilde{\mathbf{0}} .
$$

For the next two terms in (3.13), let us consider two small circles $\Omega_{a, \varepsilon_{1}}$ and $\Omega_{b, \varepsilon_{2}}$ centered at $\mathbf{a}$ and $\mathbf{b}$, respectively, such that $\Omega_{a, \varepsilon_{1}} \cap \Omega_{b, \varepsilon_{2}}=\emptyset$. Then, if $\Omega_{r}$ is a large circle centered at the origin surrounding the scatterer and the two small circles, we can write

$$
\left[\overline{\widetilde{\mathbf{u}}_{a}^{i n c}}, \widetilde{\mathbf{u}}_{b}^{s c t}\right]=\left[\overline{\widetilde{\mathbf{u}}_{a}^{i n c}}, \widetilde{\mathbf{u}}_{b}^{s c t}\right]=\left[\overline{\widetilde{\mathbf{u}}_{a}^{i n c}}, \widetilde{\mathbf{u}}_{b}^{s c t}\right]_{\Omega_{r}}-\left[\overline{\widetilde{\mathbf{u}}_{a}^{i n c}}, \widetilde{\mathbf{u}}_{b}^{s c t}\right]_{\Omega_{a, \varepsilon_{1}}}-\left[\overline{\widetilde{\mathbf{u}}_{a}^{i n c}}, \widetilde{\mathbf{u}}_{b}^{s c t}\right]_{\Omega_{b, \varepsilon_{2}}} .
$$

Since, $\overline{\widetilde{\mathbf{u}}_{a}^{i n c}}, \widetilde{\mathbf{u}}_{b}^{s c t}$ are regular solutions of the Navier equation (2.3) in the interior of $\Omega_{b, \varepsilon_{2}}$, the third integral in the right-hand side of the above relation vanishes. Now using Lemma 3.1 and letting $R \rightarrow \infty$ and $\varepsilon_{1} \rightarrow 0$, we arrive at

$$
\begin{aligned}
{\left[\widetilde{\mathbf{u}}_{a}^{i n c}, \widetilde{\mathbf{u}}_{b}^{s c t}\right]=} & \frac{1+i}{2} \sqrt{\frac{k_{p}}{\pi}} \int_{\Omega}\left(\widetilde{\mathbf{g}}_{b}^{r}(\hat{\mathbf{r}})\right)^{\top} \cdot(\hat{\mathbf{r}} \otimes \hat{\mathbf{r}}) e^{i k_{p} \mathbf{a} \cdot \hat{\mathbf{r}}} d s(\hat{\mathbf{r}}) \\
& +\frac{1+i}{2} \sqrt{\frac{k_{s}}{\pi}} \int_{\Omega}\left(\widetilde{\mathbf{g}}_{b}^{t}(\hat{\mathbf{r}})\right)^{\top} \cdot(\widetilde{\mathbf{I}}-\hat{\mathbf{r}} \otimes \hat{\mathbf{r}}) e^{i k_{s} \mathbf{a} \cdot \hat{\mathbf{r}}} d s(\hat{\mathbf{r}}) \\
& -\left(\widetilde{\mathbf{u}}_{b}^{s c t}(\mathbf{a})\right)^{\top} \cdot(\hat{\mathbf{a}} \otimes \hat{\mathbf{a}})-\frac{\lambda+\mu}{\mu}\left(\widetilde{\mathbf{u}}_{b}^{s c t}(\mathbf{a})\right)^{\top} \cdot(\widetilde{\mathbf{I}}-\hat{\mathbf{a}} \otimes \hat{\mathbf{a}}) .
\end{aligned}
$$

In a similar way and using the relation

$$
\left[\widetilde{\mathbf{u}}_{a}^{s c t}, \widetilde{\mathbf{u}}_{b}^{i n c}\right]=-{\overline{\left[\overline{\widetilde{\mathbf{u}}_{b}^{i n c}}, \widetilde{\mathbf{u}}_{a}^{s c t}\right]}}^{\top},
$$

we can find that

$$
\begin{aligned}
{\left[\overline{\widetilde{\mathbf{u}}_{a}^{s c t}}, \widetilde{\mathbf{u}}_{b}^{i n c}\right]=- } & \frac{1-i}{2} \sqrt{\frac{k_{p}}{\pi}}\left(\int_{\Omega}\left(\overline{\widetilde{\mathbf{g}}_{a}^{r}(\hat{\mathbf{r}})}\right)^{\top} \cdot(\hat{\mathbf{r}} \otimes \hat{\mathbf{r}}) e^{-i k_{s} \mathbf{b} \cdot \hat{\mathbf{r}}} d s(\hat{\mathbf{r}})\right)^{\top} \\
& -\frac{1-i}{2} \sqrt{\frac{k_{s}}{\pi}}\left(\int_{\Omega}\left(\overline{\widetilde{\mathbf{g}}_{a}^{t}(\hat{\mathbf{r}})}\right)^{\top} \cdot(\widetilde{\mathbf{I}}-\hat{\mathbf{r}} \otimes \hat{\mathbf{r}}) e^{-i k_{s} \mathbf{b} \cdot \hat{\mathbf{r}}} d s(\hat{\mathbf{r}})\right)^{\top} \\
& +\left(\left(\overline{\widetilde{\mathbf{u}}_{a}^{s c t}(\mathbf{b})}\right)^{\top} \cdot(\hat{\mathbf{b}} \otimes \hat{\mathbf{b}})\right)^{\top}+\frac{\lambda+\mu}{\mu}\left(\left(\overline{\widetilde{\mathbf{u}}_{a}^{s c t}(\mathbf{b})}\right)^{\top} \cdot(\widetilde{\mathbf{I}}-\hat{\mathbf{b}} \otimes \hat{\mathbf{b}})\right)^{\top}
\end{aligned}
$$

Up until now the first three integrals of (3.13) have been calculated; we have to deal with the last one. In view of the regularity $\overline{\widetilde{\mathbf{u}}_{a}^{s c t}}, \widetilde{\mathbf{u}}_{b}^{s c t}$ in $B_{e}$ and with the aid of Betti's 
integral theorem, the value of this integral is invariant when $\partial B_{i}$ is replaced by a circle of radius $r$ centered at the origin, which contains $\overline{B_{i}}$ in its interior. Hence,

$$
\begin{aligned}
{\left[\overline{\mathbf{u}}_{a}^{s c t}, \widetilde{\mathbf{u}}_{b}^{s c t}\right]=\left[\overline{\widetilde{\mathbf{u}}_{a}^{s c t},} \widetilde{\mathbf{u}}_{b}^{s c t}\right]_{\Omega_{r}}=} & 2 i k_{p}(\lambda+2 \mu) \int_{\Omega}\left(\widetilde{\mathbf{g}}_{b}^{r}(\hat{\mathbf{r}})\right)^{\top} \cdot \overline{\widetilde{\mathbf{g}}_{a}^{r}(\hat{\mathbf{r}})} d s(\hat{\mathbf{r}}) \\
& +2 i k_{s} \mu \int_{\Omega}\left(\widetilde{\mathbf{g}}_{b}^{t}(\hat{\mathbf{r}})\right)^{\top} \cdot \overline{\widetilde{\mathbf{g}}_{a}^{t}(\widehat{\mathbf{r}})} d s(\hat{\mathbf{r}}) .
\end{aligned}
$$

By substitution of (3.14)-(3.19) in (3.13), the assertion now easily follows.

4. Reciprocity relations. In this section the main reciprocity theorem is established. As we observe, the proof of Theorem 3.2 uses the evaluation of $\left[\widetilde{\mathbf{u}}_{a}^{t o t}, \widetilde{\mathbf{u}}_{b}^{t o t}\right]$. If, instead, we start from $\left[\widetilde{\mathbf{u}}_{a}^{\text {tot }}, \widetilde{\mathbf{u}}_{b}^{\text {tot }}\right]$, then we have the following reciprocity result.

Theorem 4.1. Consider two point-source locations $\mathbf{a}$ and $\mathbf{b}$ in the exterior $B_{e}$ of the scatterer, with $\widetilde{\mathbf{u}}_{\gamma}^{i n c}, \widetilde{\mathbf{u}}_{\gamma}^{s c t}, \gamma=a, b$, the incident and scattered fields due to the source points $\mathbf{a}$ and $\mathbf{b}$, respectively. Then for any rigid body scatterer cavity or penetrable obstacle, the following relation holds:

$$
\begin{aligned}
& \left(\widetilde{\mathbf{u}}_{b}^{s c t}(\mathbf{a})\right)^{\top} \cdot(\hat{\mathbf{a}} \otimes \hat{\mathbf{a}})+\frac{\lambda+\mu}{\mu}\left(\widetilde{\mathbf{u}}_{b}^{s c t}(\mathbf{a})\right)^{\top} \cdot(\widetilde{\mathbf{I}}-\hat{\mathbf{a}} \otimes \hat{\mathbf{a}}) \\
& =\left(\left(\widetilde{\mathbf{u}}_{a}^{s c t}(\mathbf{b})\right)^{\top} \cdot(\hat{\mathbf{b}} \otimes \hat{\mathbf{b}})\right)^{\top}+\frac{\lambda+\mu}{\mu}\left(\left(\widetilde{\mathbf{u}}_{a}^{s c t}(\mathbf{b})\right)^{\top} \cdot(\widetilde{\mathbf{I}}-\hat{\mathbf{b}} \otimes \hat{\mathbf{b}})\right)^{\top} .
\end{aligned}
$$

Proof. Using as before the relations $\widetilde{\mathbf{u}}_{\gamma}^{\text {tot }}=\widetilde{\mathbf{u}}_{\gamma}^{\text {inc }}+\widetilde{\mathbf{u}}_{\gamma}^{s c t}$, we easily obtain

$$
\left[\widetilde{\mathbf{u}}_{a}^{t o t}, \widetilde{\mathbf{u}}_{b}^{t o t}\right]=\left[\widetilde{\mathbf{u}}_{a}^{i n c}, \widetilde{\mathbf{u}}_{b}^{i n c}\right]+\left[\widetilde{\mathbf{u}}_{a}^{i n c}, \widetilde{\mathbf{u}}_{b}^{s c t}\right]+\left[\widetilde{\mathbf{u}}_{a}^{s c t}, \widetilde{\mathbf{u}}_{b}^{i n c}\right]+\left[\widetilde{\mathbf{u}}_{a}^{s c t}, \widetilde{\mathbf{u}}_{b}^{s c t}\right] .
$$

Let us first evaluate $\left[\widetilde{\mathbf{u}}_{a}^{\text {tot }}, \widetilde{\mathbf{u}}_{b}^{\text {tot }}\right]$ directly. It is obvious that for the three types of boundary conditions (see (2.9)-(2.11), we arrive at

$$
\left[\widetilde{\mathbf{u}}_{a}^{t o t}, \widetilde{\mathbf{u}}_{b}^{t o t}\right]=\widetilde{\mathbf{0}}
$$

Now following the same steps as in the proof of Theorem 3.2 we calculate each term of the right-hand side of (4.2). In particular, the first term is vanishing again, since by the third Betti's formula and the fact that $\widetilde{\mathbf{u}}_{a}^{i n c}$ and $\widetilde{\mathbf{u}}_{b}^{i n c}$ are solutions of the Navier equation (2.3) in $B_{i}$, we can obtain

$$
\left[\widetilde{\mathbf{u}}_{a}^{i n c}, \widetilde{\mathbf{u}}_{b}^{i n c}\right]=\widetilde{\mathbf{0}} .
$$

Now concerning the next two terms of (4.2), taking into account the relation $\left[\widetilde{\mathbf{u}}_{a}^{\text {sct }}, \widetilde{\mathbf{u}}_{b}^{\text {inc }}\right]=$ $-\left[\widetilde{\mathbf{u}}_{b}^{i n c}, \widetilde{\mathbf{u}}_{a}^{s c t}\right]^{\top}$, and following the same procedure as in Theorem 3.2, we end up with the relations

$$
\begin{gathered}
{\left[\widetilde{\mathbf{u}}_{a}^{i n c}, \widetilde{\mathbf{u}}_{b}^{s c t}\right]=-\left(\widetilde{\mathbf{u}}_{b}^{s t}(\mathbf{a})\right)^{\top} \cdot(\hat{\mathbf{a}} \otimes \hat{\mathbf{a}})-\frac{\lambda+\mu}{\mu}\left(\widetilde{\mathbf{u}}_{b}^{s t}(\mathbf{a})\right)^{\top} \cdot(\widetilde{\mathbf{I}}-\hat{\mathbf{a}} \otimes \hat{\mathbf{a}}),} \\
{\left[\widetilde{\mathbf{u}}_{a}^{s c t}, \widetilde{\mathbf{u}}_{b}^{i n c}\right]=\left(\left(\widetilde{\mathbf{u}}_{a}^{s c t}(\mathbf{b})\right)^{\top} \cdot(\hat{\mathbf{b}} \otimes \hat{\mathbf{b}})\right)^{\top}+\frac{\lambda+\mu}{\mu}\left(\left(\widetilde{\mathbf{u}}_{a}^{s c t}(\mathbf{b})\right)^{\top} \cdot(\widetilde{\mathbf{I}}-\hat{\mathbf{b}} \otimes \hat{\mathbf{b}})\right)^{\top} .}
\end{gathered}
$$


For the last integral of (4.2), using the fact that $\widetilde{\mathbf{u}}_{a}^{s c t}, \widetilde{\mathbf{u}}_{b}^{s c t}$ are regular solutions of the Navier equation in $B_{e}$, and with the aid of Betti's integral theorem, we arrive at

$$
\begin{aligned}
{\left[\widetilde{\mathbf{u}}_{a}^{s c t}, \widetilde{\mathbf{u}}_{b}^{s c t}\right] } & =\left[\widetilde{\mathbf{u}}_{a}^{s c t}, \widetilde{\mathbf{u}}_{b}^{s c t}\right]_{\Omega_{r}} \\
& =\int_{\Omega_{r}}\left(\left(T \widetilde{\mathbf{u}}_{b}^{s c t}(\mathbf{r})\right)^{\top} \cdot \widetilde{\mathbf{u}}_{a}^{s c t}(\mathbf{r})-\left(\widetilde{\mathbf{u}}_{b}^{s c t}(\mathbf{r})\right)^{\top} \cdot T \widetilde{\mathbf{u}}_{a}^{s c t}(\mathbf{r})\right) d s(\mathbf{r})=\widetilde{\mathbf{0}} .
\end{aligned}
$$

By substitution of (4.3)-(4.7) in (4.2) we obtain (4.1).

Furthermore, we note that, in view of (2.5), (2.8), the same reciprocity relation holds for the incident point-source field, as well as for the total exterior one.

5. Mixed scattering relations. In this section mixed scattering relations are established, relating plane-wave incidence to point-source incidence. In particular, having two point sources, we consider one of them to recede to infinity, so that we have one incident point-source field and one incident plane wave. At the end of this section, we let both point sources recede to infinity recovering known results for plane-wave incidence.

We assume an incident plane dyadic field propagating in the direction $\hat{\mathbf{d}}$, i.e.,

$$
\widetilde{\mathbf{u}}^{i n c}(\mathbf{r} ; \hat{\mathbf{d}})=(\hat{\mathbf{d}} \otimes \hat{\mathbf{d}}) e^{i k_{p} \mathbf{r} \cdot \hat{\mathbf{d}}}+(\widetilde{\mathbf{I}}-\hat{\mathbf{d}} \otimes \hat{\mathbf{d}}) e^{i k_{s} \mathbf{r} \cdot \hat{\mathbf{d}}},
$$

and recall that we have proved in Section 2 that $\lim _{a \rightarrow \infty} \widetilde{\mathbf{u}}_{a}^{i n c}(\mathbf{r})=\widetilde{\mathbf{u}}^{i n c}(\mathbf{r} ;-\hat{\mathbf{a}})$.

For an incident plane wave of the form (5.1), we denote the corresponding total field in $B_{e}$ by $\widetilde{\mathbf{u}}^{t o t}(\mathbf{r} ; \hat{\mathbf{d}})$, the scattered field by $\widetilde{\mathbf{u}}^{\text {sct }}(\mathbf{r} ; \hat{\mathbf{d}})$ and the radial and angular far-field patterns by $\widetilde{\mathbf{g}}^{r}(\hat{\mathbf{r}} ; \hat{\mathbf{d}})$ and $\widetilde{\mathbf{g}}^{t}(\hat{\mathbf{r}} ; \hat{\mathbf{d}})$, respectively. Using the relations

$$
\begin{gathered}
\lim _{a \rightarrow \infty} \widetilde{\mathbf{u}}_{a}^{s c t}(\mathbf{r})=\widetilde{\mathbf{u}}^{s c t}(\mathbf{r} ;-\hat{\mathbf{a}}), \\
\lim _{a \rightarrow \infty} \widetilde{\mathbf{g}}_{a}^{r}(\mathbf{r})=\widetilde{\mathbf{g}}^{r}(\hat{\mathbf{r}} ;-\hat{\mathbf{a}}), \quad \lim _{a \rightarrow \infty} \widetilde{\mathbf{g}}_{a}^{t}(\mathbf{r})=\widetilde{\mathbf{g}}^{t}(\hat{\mathbf{r}} ;-\hat{\mathbf{a}}),
\end{gathered}
$$

we now can establish the following.

Lemma 5.1. Let $\widetilde{\mathbf{u}}_{a}^{i n c}$ be a point source at $\mathbf{a}$. If $\widetilde{\mathbf{u}}^{i n c}(\mathbf{r} ;-\hat{\mathbf{b}})$ is an incident plane wave, then

$$
\begin{aligned}
\lim _{r \rightarrow \infty}\left[\overline{\widetilde{\mathbf{u}}_{a}^{i n c}}, \widetilde{\mathbf{u}}^{s c t}(\cdot,-\hat{\mathbf{b}})\right]_{\Omega_{r}}= & \frac{1+i}{2} \sqrt{\frac{k_{p}}{\pi}} \int_{\Omega}\left(\widetilde{\mathbf{g}}^{r}(\hat{\mathbf{r}} ;-\hat{\mathbf{b}})\right)^{\top} \cdot(\hat{\mathbf{r}} \otimes \hat{\mathbf{r}}) e^{i k_{p} \mathbf{a} \cdot \hat{\mathbf{r}}} d s(\hat{\mathbf{r}}) \\
& +\frac{1+i}{2} \sqrt{\frac{k_{s}}{\pi}} \int_{\Omega}\left(\widetilde{\mathbf{g}}^{t}(\widehat{\mathbf{r}} ;-\hat{\mathbf{b}})\right)^{\top} \cdot(\widetilde{\mathbf{I}}-\hat{\mathbf{r}} \otimes \widehat{\mathbf{r}}) e^{i k_{s} \mathbf{a} \cdot \hat{\mathbf{r}}} d s(\hat{\mathbf{r}}), \\
\lim _{\varepsilon \rightarrow 0}\left[\overline{\widetilde{\mathbf{u}}_{a}^{i n c},} \widetilde{\mathbf{u}}^{s c t}(\cdot,-\hat{\mathbf{b}})\right]_{\Omega_{a, \varepsilon}=} & \left(\widetilde{\mathbf{u}}^{s c t}(\mathbf{a} ;-\hat{\mathbf{b}})\right)^{\top} \cdot(\hat{\mathbf{a}} \otimes \hat{\mathbf{a}}) \\
& +\frac{\lambda+\mu}{\mu}\left(\widetilde{\mathbf{u}}^{s c t}(\mathbf{a} ;-\hat{\mathbf{b}})\right)^{\top} \cdot(\widetilde{\mathbf{I}}-\hat{\mathbf{a}} \otimes \hat{\mathbf{a}}) .
\end{aligned}
$$

Proof. The proof is exactly analogous as in Lemma 3.1, and so we omit it for the sake of brevity. 
We now define the dyadic plane longitudinal and transverse far-field pattern generators as follows:

$$
\begin{aligned}
\widetilde{\mathbf{G}}^{r}(\mathbf{a} ;-\hat{\mathbf{b}})= & \lim _{b \rightarrow \infty} \widetilde{\mathbf{G}}_{b}^{r}(\mathbf{a}) \\
= & \frac{(1-i) \sqrt{k_{p}}}{4} \int_{\Omega}\left(\widetilde{\mathbf{g}}^{r}(\hat{\mathbf{r}} ;-\hat{\mathbf{b}})\right)^{\top} \cdot(\hat{\mathbf{r}} \otimes \hat{\mathbf{r}}) e^{i k_{p} \mathbf{a} \cdot \hat{\mathbf{r}}} d s(\hat{\mathbf{r}}) \\
& +\frac{i \sqrt{\pi}}{2}\left(\widetilde{\mathbf{u}}^{s c t}(\mathbf{a} ;-\hat{\mathbf{b}})\right)^{\top} \cdot(\hat{\mathbf{a}} \otimes \hat{\mathbf{a}}), \\
\widetilde{\mathbf{G}}^{t}(\mathbf{a} ;-\hat{\mathbf{b}})= & \lim _{b \rightarrow \infty} \widetilde{\mathbf{G}}_{b}^{t}(\mathbf{a}) \\
= & \frac{(1-i) \sqrt{k_{s}}}{4} \int_{\Omega}\left(\widetilde{\mathbf{g}}^{t}(\hat{\mathbf{r}} ;-\hat{\mathbf{r}})\right)^{\top} \cdot(\widetilde{\mathbf{I}}-\hat{\mathbf{r}} \otimes \hat{\mathbf{r}}) e^{i k_{s} \mathbf{a} \cdot \hat{\mathbf{r}}} d s(\hat{\mathbf{r}}) \\
& +\frac{i \sqrt{\pi}}{2} \frac{\lambda+\mu}{\mu}\left(\widetilde{\mathbf{u}}^{s c t}(\mathbf{a} ;-\hat{\mathbf{b}})\right)^{\top} \cdot(\widetilde{\mathbf{I}}-\hat{\mathbf{a}} \otimes \hat{\mathbf{a}}),
\end{aligned}
$$

where the far-field pattern generators $\widetilde{\mathbf{G}}_{b}^{r}(\mathbf{a})$ and $\widetilde{\mathbf{G}}_{b}^{t}(\mathbf{a})$ are defined by (3.10) and (3.11), respectively.

THEOREM 5.2. If $\widetilde{\mathbf{u}}_{a}^{i n c}$ and $\widetilde{\mathbf{u}}_{b}^{i n c}$ are two incident point-source fields, then the following relations hold:

$$
\begin{aligned}
\lim _{a \rightarrow \infty} \widetilde{\mathbf{G}}_{b}^{r}(\mathbf{a}) & =\frac{e^{i k_{p} a}}{2} \sqrt{\frac{\pi}{a}}\left(\widetilde{\mathbf{g}}_{b}^{r}(-\hat{\mathbf{a}})\right)^{\top} \cdot(\hat{\mathbf{a}} \otimes \hat{\mathbf{a}}), \\
\lim _{a \rightarrow \infty} \widetilde{\mathbf{G}}_{b}^{t}(\mathbf{a}) & =\frac{e^{i k_{s} a}}{2} \sqrt{\frac{\pi}{a}}\left(\widetilde{\mathbf{g}}_{b}^{t}(-\hat{\mathbf{a}})\right)^{\top} \cdot(\widetilde{\mathbf{I}}-\hat{\mathbf{a}} \otimes \hat{\mathbf{a}}), \\
\lim _{a \rightarrow \infty} \widetilde{\mathbf{G}}^{r}(\mathbf{a} ;-\hat{\mathbf{b}}) & =\frac{e^{i k_{p} a}}{2} \sqrt{\frac{\pi}{a}}\left(\widetilde{\mathbf{g}}^{r}(-\hat{\mathbf{a}} ;-\hat{\mathbf{b}})\right)^{\top} \cdot(\hat{\mathbf{a}} \otimes \hat{\mathbf{a}}), \\
\lim _{a \rightarrow \infty} \widetilde{\mathbf{G}}^{t}(\mathbf{a} ;-\hat{\mathbf{b}}) & =\frac{e^{i k_{s} a}}{2} \sqrt{\frac{\pi}{a}}\left(\widetilde{\mathbf{g}}^{t}(-\hat{\mathbf{a}} ;-\hat{\mathbf{b}})\right)^{\top} \cdot(\widetilde{\mathbf{I}}-\hat{\mathbf{a}} \otimes \hat{\mathbf{a}}) .
\end{aligned}
$$

Proof. First of all, let us prove Eq. (5.8). Using (2.13) and (3.10), we can obtain the following:

$$
\begin{aligned}
\lim _{a \rightarrow \infty} \widetilde{\mathbf{G}}_{b}^{r}(\mathbf{a})= & \frac{(1-i) \sqrt{k_{p}}}{4} \lim _{a \rightarrow \infty} \int_{\Omega}\left(\widetilde{\mathbf{g}}_{b}^{r}(\hat{\mathbf{r}})\right)^{\top} \cdot(\hat{\mathbf{r}} \otimes \hat{\mathbf{r}}) e^{i k_{p} \mathbf{a} \cdot \hat{\mathbf{r}}} d s(\hat{\mathbf{r}}) \\
& +\frac{i \sqrt{\pi}}{2}\left(\widetilde{\mathbf{g}}_{b}^{r}(\hat{\mathbf{a}})\right)^{\top} \cdot(\hat{\mathbf{a}} \otimes \hat{\mathbf{a}}) \frac{e^{i k_{p} a}}{\sqrt{a}} .
\end{aligned}
$$

We now want to find the limit of the integral in (5.12). Without loss of generality, we choose polar coordinates so that the point source at a will be on the $y$-axis. Then we note that for $\theta=\pi / 2$ we have $\hat{\mathbf{r}}=\hat{\mathbf{a}}$, while for $\theta=3 \pi / 2$ we have $\hat{\mathbf{r}}=-\hat{\mathbf{a}}$. We break the above integral in (5.12) into a sum of two integrals,

$$
\begin{aligned}
\int_{\Omega}\left(\widetilde{\mathbf{g}}_{b}^{r}(\hat{\mathbf{r}})\right)^{\top} \cdot(\hat{\mathbf{r}} \otimes \hat{\mathbf{r}}) e^{i k_{p} \mathbf{a} \cdot \hat{\mathbf{r}}} d s(\hat{\mathbf{r}})= & \int_{0}^{\pi}\left(\widetilde{\mathbf{g}}_{b}^{r}(\hat{\mathbf{r}})\right)^{\top} \cdot(\hat{\mathbf{r}} \otimes \hat{\mathbf{r}}) e^{i k_{p} \mathbf{a} \cdot \hat{\mathbf{r}}} d s(\hat{\mathbf{r}}) \\
& +\int_{\pi}^{2 \pi}\left(\widetilde{\mathbf{g}}_{b}^{r}(\hat{\mathbf{r}})\right)^{\top} \cdot(\hat{\mathbf{r}} \otimes \hat{\mathbf{r}}) e^{i k_{p} \mathbf{a} \cdot \hat{\mathbf{r}}} d s(\hat{\mathbf{r}}),
\end{aligned}
$$


each containing one stationary point, i.e., $\theta=\pi / 2$ and $\theta=3 \pi / 2$ for the intervals $[0, \pi]$ and $[\pi, 2 \pi]$, respectively. Applying the method of stationary phase to each of these integrals $[9$, p. 254] and summing the results, we get

$$
\begin{aligned}
& \lim _{a \rightarrow \infty} \int_{\Omega}\left(\widetilde{\mathbf{g}}_{b}^{r}(\hat{\mathbf{r}})\right)^{\top} \cdot(\hat{\mathbf{r}} \otimes \hat{\mathbf{r}}) e^{i k_{p} \mathbf{a} \cdot \hat{\mathbf{r}}} d s(\hat{\mathbf{r}}) \\
& =\sqrt{\frac{\pi}{k_{p} a}}(1-i) e^{i k_{p} a}\left(\widetilde{\mathbf{g}}_{b}^{r}(\hat{\mathbf{a}})\right)^{\top} \cdot(\hat{\mathbf{a}} \otimes \hat{\mathbf{a}})+\frac{e^{i k_{p} a}}{2} \sqrt{\frac{\pi}{a}}\left(\widetilde{\mathbf{g}}_{b}^{r}(-\hat{\mathbf{a}})\right)^{\top} \cdot(\hat{\mathbf{a}} \otimes \hat{\mathbf{a}}) .
\end{aligned}
$$

Combining the above relation with (5.12), the assertion of the theorem follows. With similar arguments as before we can establish (5.9), (5.10) and (5.11).

In what follows we formulate the next basic theorem, which can be proved following the same steps as in the general scattering theorem (Theorem 3.2).

Theorem 5.3. Assume two point-source locations $\mathbf{a}$ and $\mathbf{b}$ in $B_{e}$. If we let $b \rightarrow \infty$, then we have

$$
\begin{aligned}
& \widetilde{\mathbf{G}}^{r}(\mathbf{a} ; \hat{\mathbf{b}})+\left(\overline{\widetilde{\mathbf{g}}_{a}^{r}(-\hat{\mathbf{b}})}\right)^{\top}+\widetilde{\mathbf{G}}^{t}(\mathbf{a} ; \hat{\mathbf{b}})+\left(\overline{\widetilde{\mathbf{g}}_{a}^{t}(-\hat{\mathbf{b}})}\right)^{\top} \\
= & -k_{p}(\lambda+2 \mu) \sqrt{\pi} \int_{\Omega}\left(\widetilde{\mathbf{g}}^{r}(\hat{\mathbf{r}} ;-\hat{\mathbf{b}})\right)^{\top} \cdot \overline{\widetilde{\mathbf{g}}_{a}^{r}(\hat{\mathbf{r}})} d s(\hat{\mathbf{r}}) \\
& -k_{s} \mu \sqrt{\pi} \int_{\Omega}\left(\widetilde{\mathbf{g}}^{t}(\hat{\mathbf{r}} ;-\hat{\mathbf{b}})\right)^{\top} \cdot \overline{\widetilde{\mathbf{g}}_{a}^{t}(\hat{\mathbf{r}})} d s(\hat{\mathbf{r}}) .
\end{aligned}
$$

The following mixed reciprocity principle, due to an incident point-source field and an incident plane field, is established in the next theorem.

THEOREM 5.4. If $\widetilde{\mathbf{u}}_{a}^{i n c}$ is an incident point-source wave field and $\widetilde{\mathbf{u}}^{i n c}(\mathbf{r} ;-\hat{\mathbf{b}})$ an incident plane wave propagating in the direction $-\hat{\mathbf{b}}$, then the following relation holds:

$$
\begin{aligned}
& \left(\widetilde{\mathbf{u}}^{s c t}(\mathbf{a} ;-\hat{\mathbf{b}})\right)^{\top} \cdot(\hat{\mathbf{a}} \otimes \hat{\mathbf{a}})+\frac{\lambda+\mu}{\mu}\left(\widetilde{\mathbf{u}}^{s c t}(\mathbf{a} ;-\hat{\mathbf{b}})\right)^{\top} \cdot(\widetilde{\mathbf{I}}-\hat{\mathbf{a}} \otimes \hat{\mathbf{a}}) \\
& =\left(\left(\widetilde{\mathbf{g}}_{a}^{r}(\hat{\mathbf{b}})\right)^{\top} \frac{e^{i k_{p} b}}{\sqrt{b}} \cdot(\hat{\mathbf{b}} \otimes \hat{\mathbf{b}})\right)^{\top}+\frac{\lambda+\mu}{\mu}\left(\left(\widetilde{\mathbf{g}}_{a}^{t}(\hat{\mathbf{b}})\right)^{\top} \frac{e^{i k_{s} b}}{\sqrt{b}} \cdot(\widetilde{\mathbf{I}}-\hat{\mathbf{b}} \otimes \hat{\mathbf{b}})\right)^{\top} .
\end{aligned}
$$

Proof. The assertion easily follows with the aid of (2.13) and (5.2), by letting in (4.1), $b \rightarrow \infty$.

We end this section by combining relations (5.3) and (5.6)-(5.11). Hence,

$$
\begin{aligned}
\lim _{a \rightarrow \infty} \lim _{b \rightarrow \infty} \widetilde{\mathbf{G}}_{b}^{r}(\mathbf{a}) & =\lim _{b \rightarrow \infty} \lim _{a \rightarrow \infty} \widetilde{\mathbf{G}}_{b}^{r}(\mathbf{a}) \\
& =\frac{e^{i k_{p} a}}{2} \sqrt{\frac{\pi}{a}}\left(\widetilde{\mathbf{g}}^{r}(-\hat{\mathbf{a}} ;-\hat{\mathbf{b}})\right)^{\top} \cdot(\hat{\mathbf{a}} \otimes \hat{\mathbf{a}})
\end{aligned}
$$

and

$$
\begin{aligned}
\lim _{a \rightarrow \infty} \lim _{b \rightarrow \infty} \widetilde{\mathbf{G}}_{b}^{t}(\mathbf{a}) & =\lim _{b \rightarrow \infty} \lim _{a \rightarrow \infty} \widetilde{\mathbf{G}}_{b}^{t}(\mathbf{a}) \\
& =\frac{e^{i k_{s} a}}{2} \sqrt{\frac{\pi}{a}}\left(\widetilde{\mathbf{g}}^{t}(-\hat{\mathbf{a}} ;-\hat{\mathbf{b}})\right)^{\top} \cdot(\widetilde{\mathbf{I}}-\hat{\mathbf{a}} \otimes \hat{\mathbf{a}}) .
\end{aligned}
$$


Let us note that when both point sources recede to infinity, then from (5.15) and (5.16), one can get the known scattering theorems for plane-wave scattering. Analogous properties for acoustics and electromagnetics can be found in [5, 7, while for 3D-elasticity in [13.

6. The optical theorem. In this section an expression for the scattering cross section, due to an incident point-source field, will be established. Following a similar analysis as the one for the 3D-case in [14-17, the energy flux vector for the scattered field in the radiation zone is given by

$$
\mathbf{P}_{a}^{s c t}(\mathbf{r})=\widetilde{\mathbf{E}}_{a}^{s c t}(\mathbf{r}): \mathbf{c} \otimes \mathbf{c},
$$

where $\widetilde{\widetilde{\mathbf{E}}}_{a}^{s c t}$ denotes the energy triadic defined by

$$
\widetilde{\mathbf{E}}_{a}^{s c t}(\mathbf{r})=\omega \Im\left[\left({\overline{\widetilde{\mathbf{u}}_{a}^{s c t}(\mathbf{r})}}^{\top} \cdot \widetilde{\widetilde{\mathbf{S}}}_{a}^{s c t}(\mathbf{r})\right)^{213}\right],
$$

where $\mathbf{c}$ is an arbitrary constant vector. The scattered field $\widetilde{\mathbf{u}}_{a}^{s c t}(\mathbf{r})$ due to the incident point-source field at $\mathbf{a}$, generates the stress triadic $\widetilde{\widetilde{\mathbf{S}}}_{a}^{s c t}(\mathbf{r})$ given by the relation

$$
\widetilde{\mathbf{S}}_{a}^{s c t}(\mathbf{r})=\lambda \widetilde{\mathbf{I}} \otimes\left(\nabla_{\mathbf{r}} \cdot \widetilde{\mathbf{u}}_{a}^{s c t}(\mathbf{r})\right)+\mu \nabla_{\mathbf{r}} \otimes \widetilde{\mathbf{u}}_{a}^{s c t}(\mathbf{r})+\mu\left(\nabla_{\mathbf{r}} \otimes \widetilde{\mathbf{u}}_{a}^{s c t}(\mathbf{r})\right)^{213},
$$

where 213 denotes the order of the tensorial product in the corresponding triadic.

Following the same proceedure as in [15], for $r \rightarrow \infty$, we evaluate the quantities $\nabla_{\mathbf{r}} \cdot \widetilde{\mathbf{u}}_{a}^{s c t}(\mathbf{r})$ and $\nabla_{\mathbf{r}} \otimes \widetilde{\mathbf{u}}_{a}^{s c t}(\mathbf{r})$ of (6.3). After lengthy calculations, we obtain the following:

$$
\begin{aligned}
\widetilde{\mathbf{S}}_{a}^{s c t}(\mathbf{r})= & i k_{p}(\lambda \widetilde{\mathbf{I}} \otimes \hat{\mathbf{r}}+2 \mu \hat{\mathbf{r}} \otimes \widetilde{\mathbf{I}}) \cdot \widetilde{\mathbf{g}}_{a}^{r}(\hat{\mathbf{r}}) \frac{e^{i k_{p} r}}{\sqrt{r}} \\
& +i k_{s}\left[\mu \hat{\mathbf{r}} \otimes \widetilde{\mathbf{g}}_{a}^{t}(\hat{\mathbf{r}})+\mu\left(\hat{\mathbf{r}} \otimes \widetilde{\mathbf{g}}_{a}^{t}(\hat{\mathbf{r}})\right)^{213}\right] \frac{e^{i k_{s} r}}{\sqrt{r}}+O\left(r^{-3 / 2}\right) .
\end{aligned}
$$

The energy flux of the scattered wave at point $\hat{\mathbf{r}}$ in the direction of observation, with the aid of (6.1) and (6.2), is given by

$$
\hat{\mathbf{r}} \cdot \mathbf{P}_{a}^{s c t}(\mathbf{r})=\hat{\mathbf{r}} \cdot \omega \Im\left[\left({\overline{\widetilde{\mathbf{u}}_{a}^{s c t}(\mathbf{r})}}^{\top} \cdot \widetilde{\widetilde{\mathbf{S}}}_{a}^{s c t}(\mathbf{r})\right)^{213}\right]: \mathbf{c} \otimes \mathbf{c} .
$$

Hence, in view of (2.13), after lengthy calculations, and using the property of the left transpose of a triadic [8], we obtain the following, as $r \rightarrow \infty$ :

$$
\hat{\mathbf{r}} \cdot \widetilde{\widetilde{\mathbf{E}}}_{a}^{s c t}(\mathbf{r})=\frac{\rho}{r} \Re\left(c_{p}^{3} k_{p}^{2}{\overline{\widetilde{\mathbf{g}}_{a}^{r}(\hat{\mathbf{r}})}}^{\top} \cdot \widetilde{\mathbf{g}}_{a}^{r}(\hat{\mathbf{r}})+c_{s}^{3} k_{s}^{2}{\overline{\widetilde{\mathbf{g}}_{a}^{t}(\hat{\mathbf{r}})}}^{\top} \cdot \widetilde{\mathbf{g}}_{a}^{t}(\hat{\mathbf{r}})\right)+O\left(r^{-3 / 2}\right) .
$$

By substitution of (6.6) in (6.5), the outward energy flux at the point $\mathbf{r}$ in the direction $\hat{\mathbf{r}}$ is given by

$$
\hat{\mathbf{r}} \cdot \mathbf{P}_{a}^{s c t}(\mathbf{r})=\frac{\rho \omega^{3}}{r}\left(\frac{1}{k_{p}}\left\|\widetilde{\mathbf{g}}_{a}^{r}(\hat{\mathbf{r}}) \cdot \mathbf{c}\right\|^{2}+\frac{1}{k_{s}}\left\|\widetilde{\mathbf{g}}_{a}^{t}(\hat{\mathbf{r}}) \cdot \mathbf{c}\right\|^{2}\right)+O\left(r^{-3 / 2}\right), \quad r \rightarrow \infty .
$$


In what follows, we deal with the energy triadic and the energy flux vector for the incident point-source field in order to define the corresponding differential scattering cross section $\sigma_{a}^{s c t}$.

Following the same steps as before and using (3.7), after some computational effort, the stress triadic generated by $\widetilde{\mathbf{u}}_{a}^{i n c}$ is given by

$$
\begin{aligned}
\widetilde{\widetilde{S}}_{a}^{i n c}(\mathbf{r})= & -\frac{i k_{p}}{4(\lambda+2 \mu)} H_{1}^{(1)}\left(k_{p} \varepsilon\right)((\lambda \widetilde{\mathbf{I}}+2 \mu \hat{\mathbf{R}} \otimes \hat{\mathbf{R}}) \otimes \hat{\mathbf{R}}) \\
& -\frac{i k_{s}}{4} H_{1}^{(1)}\left(k_{s} \varepsilon\right)\left[\hat{\mathbf{R}} \otimes \widetilde{\mathbf{I}}+(\hat{\mathbf{R}} \otimes \widetilde{\mathbf{I}})^{213}-2 \hat{\mathbf{R}} \otimes \hat{\mathbf{R}} \otimes \hat{\mathbf{R}}\right] \\
& +\frac{i}{4 \varepsilon \mu(\lambda+2 \mu)}\left(\mu H_{2}^{(1)}\left(k_{p} \varepsilon\right)-(\lambda+2 \mu) H_{2}^{(1)}\left(k_{s} \varepsilon\right)\right) \\
& \times\left[\lambda \widetilde{\mathbf{I}} \otimes \widetilde{\hat{\mathbf{R}}}-\mu \hat{\mathbf{R}} \otimes \widetilde{\mathbf{I}}-\mu(\hat{\mathbf{R}} \otimes \widetilde{\mathbf{I}})^{213}+4 \mu \hat{\mathbf{R}} \otimes \hat{\mathbf{R}} \otimes \hat{\mathbf{R}}\right]
\end{aligned}
$$

where " $\times$ " denotes standard multiplication. Then, the corresponding energy triadic is given by the relation

$$
\widetilde{\mathbf{E}}_{a}^{i n c}(\mathbf{r})=\omega \Im\left[\left({\overline{\widetilde{\mathbf{u}}_{a}^{i n c}(\mathbf{r})}}^{\top} \cdot \widetilde{\widetilde{\mathbf{S}}}_{a}^{i n c}(\mathbf{r})\right)^{213}\right] .
$$

Then, finding the asymptotic form of $\hat{\mathbf{r}} \cdot \widetilde{\widetilde{\mathbf{E}}}_{a}^{i n c}$ for $r \rightarrow \infty$, lengthy calculations lead to the normal energy flux of the incident field in the radial direction far away from the scatterer being given by

$$
\hat{\mathbf{r}} \cdot \mathbf{P}_{a}^{i n c}(\mathbf{r})=\frac{1}{r} \frac{\omega}{8 \pi \rho}\left[\frac{\hat{\mathbf{r}} \otimes \hat{\mathbf{r}}}{c_{p}^{2}}+\frac{\widetilde{\mathbf{I}}-\hat{\mathbf{r}} \otimes \hat{\mathbf{r}}}{c_{s}^{2}}\right]: \mathbf{c} \otimes \mathbf{c}+O\left(r^{-3 / 2}\right), \quad r \rightarrow \infty .
$$

Following an analogous procedure as in [13, we are ready now to define the differential scattering cross section due to a point source at a as follows:

$$
\sigma(\hat{\mathbf{r}})=\lim _{r \rightarrow \infty} \frac{2 \pi r \hat{\mathbf{r}} \cdot \mathbf{P}_{a}^{i n c}(\mathbf{r})}{\int_{\Omega_{r}} \hat{\mathbf{r}} \cdot \mathbf{P}_{a}^{i n c}(\mathbf{r}) d s(\mathbf{r})}
$$

where $\hat{\mathbf{r}}$ is the direction of observation, the product $\hat{\mathbf{r}} \cdot \mathbf{P}_{a}^{i n c}(\mathbf{r})$ describes the normal energy flux of the incident point-source field in the radial direction, while the integral in the denominator is the total energy flux in all directions.

Combining relations (6.10) and (6.11), and after some calculations, we find for the rigid scatterer case the following:

$$
\sigma(\hat{\mathbf{r}})=16 \pi \omega \frac{c_{p}\left\|\widetilde{\mathbf{g}}_{a}^{r}(\hat{\mathbf{r}}) \cdot \mathbf{c}\right\|^{2}+c_{s}\left\|\widetilde{\mathbf{g}}_{a}^{t}(\hat{\mathbf{r}}) \cdot \mathbf{c}\right\|^{2}}{\left(c_{p}^{-2}+c_{s}^{-2}\right)\|\mathbf{c}\|^{2}} .
$$

If now we take the integral for $\sigma(\hat{\mathbf{r}})$ over the unit circle $\Omega$, then we can define the scattering cross section or total cross section, which is a measure of the disturbance 
caused by the scatterer to the propagation of the incident point-source field, i.e.,

$$
\sigma_{a}^{s c t}=\frac{1}{2 \pi} \int_{\Omega} \sigma(\hat{\mathbf{r}}) d s(\hat{\mathbf{r}})
$$

hence, with the aid of (6.12),

$$
\sigma_{a}^{s c t}=\frac{8 \omega}{\left(c_{p}^{-2}+c_{s}^{-2}\right)\|\mathbf{c}\|^{2}} \int_{\Omega}\left(c_{p}\left\|\widetilde{\mathbf{g}}_{a}^{r}(\hat{\mathbf{r}}) \cdot \mathbf{c}\right\|^{2}+c_{s}\left\|\widetilde{\mathbf{g}}_{a}^{t}(\hat{\mathbf{r}}) \cdot \mathbf{c}\right\|^{2}\right) d s(\hat{\mathbf{r}}) .
$$

In addition, if we set $\mathbf{a}=\mathbf{b}$ in Theorem 3.2, contract (3.12) with a constant vector $\mathbf{c}$ from the left, and then from the right, we can obtain the following:

$$
\begin{aligned}
& 2 \Re\left[\mathbf{c} \cdot \widetilde{\mathbf{G}}_{a}^{r}(\mathbf{a}) \cdot \mathbf{c}\right]+2 \Re\left[\mathbf{c} \cdot \widetilde{\mathbf{G}}_{a}^{t}(\mathbf{a}) \cdot \mathbf{c}\right] \\
& =-k_{p}(\lambda+2 \mu) \sqrt{\pi} \int_{\Omega}\left\|\widetilde{\mathbf{g}}_{a}^{r}(\widehat{\mathbf{r}}) \cdot \mathbf{c}\right\|^{2} d s(\hat{\mathbf{r}})-k_{s} \mu \sqrt{\pi} \int_{\Omega}\left\|\widetilde{\mathbf{g}}_{a}^{t}(\widehat{\mathbf{r}}) \cdot \mathbf{c}\right\|^{2} d s(\hat{\mathbf{r}}) .
\end{aligned}
$$

So, taking into account (6.14) and (6.15), we can obtain for the rigid scatterer case, the following theorem:

TheOREm 6.1. For a point source at $\mathbf{a}$, and an arbitrary constant vector $\mathbf{c}$, we have

$$
\sigma_{a}^{s c t}=\frac{8}{\left(c_{p}^{-2}+c_{s}^{-2}\right)\|\mathbf{c}\|^{2}} \times\left[-2 \Re\left(\mathbf{c} \cdot \widetilde{\mathbf{G}}_{a}^{r}(\mathbf{a}) \cdot \mathbf{c}\right)-2 \Re\left(\mathbf{c} \cdot \widetilde{\mathbf{G}}_{a}^{t}(\mathbf{a}) \cdot \mathbf{c}\right)\right] .
$$

REMARK 6.2. This theorem is called the Optical Theorem or, often, the forward scattering theorem. Furthermore, following a similar procedure to the above, we can obtain the optical theorem for the cases of the cavity, or the penetrable obstacle.

\section{REFERENCES}

[1] T. Arens, Existence of solution in elastic wave scattering by unbounded rough surfaces. Math. Methods Appl. Sci. 25 (2002), 507-528. MR1895657 (2004a:74036)

[2] T. Arens, Linear sampling methods for $2 D$ inverse elastic wave scattering. Inverse Problems $\mathbf{1 7}$ (2001), 1445-1464. MR.1862201 (2002g:35207)

[3] T. Arens, Uniqueness for elastic wave scattering by rough surfaces. SIAM J. Math. Anal. 33 (2001), 461-476. MR 1857980 (2002f:74037)

[4] C. Athanasiadis, P. A. Martin and I. G. Stratis, On spherical-wave scattering by a spherical scatterer and related near-field inverse problems. IMA J. Appl. Math. 66 (2001), 539-549. MR1869404 (2002i:35141)

[5] C. Athanasiadis, P. A. Martin, A. Spyropoulos and I. G. Stratis, Scattering relations for point sources: Acoustic and electromagnetic waves. J. Math. Phys. 43 (2002), 5683-5697. MR1936544 (2003k:78012)

[6] C. Athanasiadis, P. A. Martin and I. G. Stratis, On the scattering of point-generated electromagnetic waves by a perfectly conducting sphere, and related near-field inverse problems. Z. Angew. Math. Mech. 83 (2003), 129-136. MR.1960116 (2003k:78013)

[7] C. Athanasiadis and N. Berketis, Scattering relations for point-source excitation in chiral media. Math. Methods Appl. Sci. 29 (2006), 27-48. MR2185632 (2006j:78008)

[8] A. Ben-Menahem and S. J. Singh, Seismic Waves and Sources, Springer-Verlag, New York, 1981.

[9] D. Colton, Partial Differential Equations, Random House/Birkhäuser Mathematics Series, New York, 1988. MR0943346 (89j:35002)

[10] D. Colton and R. Kress, Inverse Acoustic and Electromagnetic Scattering Theory, Springer-Verlag, Berlin, 1992. MR.1183732 (93j:35124) 
[11] G. Dassios, K. Kiriaki and D. Polyzos, On the scattering amplitudes for elastic waves. Z. Angew. Math. Phys. 38 (1987), 856-873. MR0928588 (89a:73019)

[12] G. Dassios, K. Kiriaki and D. Polyzos, Scattering theorems for complete dyadic fields. Int. J. Eng. Sci. 33 (1995), 269-277. MR.1311563 (95i:76092)

[13] G. Dassios and R. Kleinman, Low Frequency Scattering, Clarendon Press, Oxford, 2000. MR1858914 (2003a:35001)

[14] G. Dassios, Energy functionals in scattering theory and inversion of low frequency moments. In "Advanced Course in Wavefield Inversion", International Centre for Mechanical Sciences 398, ed. A. Wirgin; Udine, (2000), 1-58.

[15] G. Dassios and K. Karveli, Dyadic scattering by small obstacles: The rigid sphere. Quart. J. Mech. Appl. Math. 54 (2001), 371-374. MR1846998(2002e:74022)

[16] G. Dassios and K. Karveli, Scattering of a spherical dyadic field by a small rigid sphere. Mathematics and Mechanics of Solids. 7 (2002), 3-40. MR1900932 (2003a:74031)

[17] G. Dassios, K. Karveli, S. Kattis and N. Kathreptas, The disturbance of a plane dyadic wave by a small spherical cavity. Int. J. Eng. Science 40 (2002), 1975-2000. MR 1935524 (2003h:74040)

[18] V. D. Kupradze, Potential Methods in the Theory of Elasticity, Israel Program for Scientific Translations, Jerusalem, 1965. MR0223128 (36:6177)

[19] P. A. Martin, On the scattering of elastic waves by an elastic inclusion in two dimensions. Quart. J. Mech. Appl. Math. 43 (1990), 275-291. MR.1070957 (92a:73020)

[20] P. M. Morse and H. Feshbach, Methods of Theoretical Physics, Vols. I, II, McGraw-Hill, New York, 1953. MR0059774 (15:583h)

[21] R. G. Payton, Two-dimensional anisotropic elastic waves emanating from a point source. Proc. Cambridge Philos. Soc. 70 (1971), 191-210. MR0293891 (45:2967)

[22] R. G. Payton, Two dimensional wave front shape induced in a homogeneously strained elastic body by a point perturbing body force. Arch. Rational Mech. Anal. 32 (1969), 311-330. MR0235781 (38:4084)

[23] G. Pelekanos and V. Sevroglou, Inverse scattering by penetrable objects in two-dimensional elastodynamics. J. Comput. Appl. Math. 151 (2003), 129-140. MR.1950233 (2003j:35326)

[24] O. Poisson, Calculs des pôles de résonance associés à la diffraction d'ondes acoustiques et élastiques en dimension 2, Thèse, Université de Paris IX (Paris-Dauphine), Paris, 1992.

[25] R. Potthast, Point Sources and Multipoles in Inverse Scattering Theory, Chapman and Hall/CRC, Boca Raton, FL, 2001. MR 1853728 (2002j:35313)

[26] V. Sevroglou and G. Pelekanos, An inversion algorithm in two-dimensional elasticity. J. Math. Anal. Appl. 263 (2001), 277-293. MR1865281 (2002g:74030)

[27] V. Sevroglou and G. Pelekanos. Two-dimensional elastic Herglotz functions and their applications in inverse scattering. Journal of Elasticity 68 (2002), 123-144. MR2024308 (2005b:74018)

[28] V. Sevroglou, The far-field operator for penetrable and absorbing obstacles in $2 D$ inverse elastic scattering. Inverse Problems 21 (2005), 717-738. MR.2146285 (2006a:74042)

[29] C. T. Tai, Dyadic Green Functions in Electromagnetic Theory, IEEE Press Series on Electromagnetic Waves, Piscataway, NJ, 1994. MR.1420621 (97k:78015)

[30] V. Twersky, Multiple scattering of electromagnetic waves by arbitary configurations. J. Math. Eng. Sci. 8 (1967), 589-610.

[31] C.-Y. Wang, Elastic fields produced by a point source in solids of general anisotropy. J. Engrg. Math. 32 (1997), 41-52. MR.1474609 (98d:73019) 